\title{
Study of Clustered Energy Saving Routing Protocol for Mobile Nodes in Three-dimensional Space
}

\author{
Cheng Huang, Run Zhang, Xiao-bei Wu and Zhi-liang Xu \\ School of Automation, Nanjing University of Science and Technology, Nanjing, \\ China \\ E-mail: hearthc@163.com
}

\begin{abstract}
Movement of sensor nodes may cause network topology change, communication link fracture and signal propagation delay, while increasing network energy consumption and shortening lifetime of the whole network. This paper proposes an energy-efficient clustering routing algorithm based on mobile wireless sensor networks in three-dimensional space. The algorithm uses multiple-hop routing mechanism, which can effectively balance the node energy consumption in network. The simulation results show that the algorithm has a good effect on node energy saving in the process of moving at different speeds. Thus the network always has more residual energy and longer survival time, which can effectively balance the energy consumption of wireless sensor network and extend the network lifetime.
\end{abstract}

Keywords: Mobile network; Multi-hop; Clustering; Energy Saving

\section{Introduction}

Mobile wireless sensor networks are capable of sensing, processing, mobile, communication and other functions [1], can effectively improve the network throughput and connectivity. But the mobile nodes will lead to corresponding network topology changes comparing to static network[4-5].The traditional static routing protocol often results in delay or loss of data transmission and wasting too much energy in nodes when it is applied to mobile wireless sensor networks. With the continuous development and the in-depth study of mobile sensor technology, the mobile sensor networks will be widely used in various research areas, with the standard of faster, more efficient, less energy for people to deliver data and information [6].

Some of current wireless sensor network researches have a typical three-dimensional topology, such as a wide range of environmental monitoring in forests, which is three-dimensional network topology [7]. The design of three-dimensional network routing brings new challenges, and it is necessary to ensure efficient and accurate communication in three-dimensional space. The study of three-dimensional routing protocol has a wide application prospect [8-9].

This paper presents a node mobility energy efficient routing protocol based on lumping of three-dimensional (3D-NMEERPL). Combining the features of energy balance based on wireless sensor network clustering algorithm [10], 3D-NMEERPL algorithm uses the idea of clustering, and considers the number of the optical cluster head, node residual energy, the distance between nodes and the base station, the distribution density of nodes, etc, to make sure that the cluster head is more reasonable and the network energy consumption is more balanced at the stage of the setup of cluster. 


\section{3D-NMEERPL Mobility Model and Energy Model}

\subsection{Mobility Model}

Sensor nodes are able to move in three dimensions randomly, and they are applicable to random mobility model[11] and passive mobility model, the energy consumed by the power sector in the process of moving would not be considered in this article. Normalizing the time reference, nodes mobility meet the following two rules:

(1) The moving speed of node is $V$, each node moves in steps of $V \cdot 1=V$. The coordinate of node is $(x, y, z)$ at initial time. The coordinate will became $(x+V \cdot \sin \theta \cos \phi, y+V \cdot \sin \theta \sin \phi, z+V \cdot \cos \theta)$ after moving one step randomly; where $0 \leq \theta \leq \pi, 0 \leq \phi \leq 2 \pi$.

(2) Select the next direction of movement randomly when a node meet boundary.

\subsection{Energy Model}

In order to analyze and study the performance of 3D-NMEERPL protocol algorithm conveniently, we use the first radio model in terms of energy consumption in paper[12], shown in Figure 1.

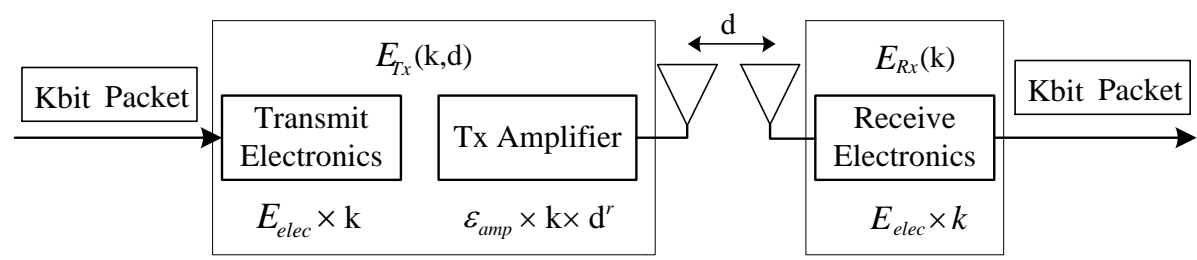

Figure 1. The First Order of Radio Energy Model Diagram

Assuming the channel is two-way symmetrical, when the sender sends K-bit data to the recipient, the energy consumption is:

$$
\begin{gathered}
E_{T X}(k, d)=E_{T X-\text { elec }}(k)+E_{T X-a m p}(k, d)= \begin{cases}E_{\text {elec }} \cdot k+\mu_{f s} \cdot k \cdot d^{2} & , d<d_{0} \\
E_{\text {elec }} \cdot k+\mu_{m p} \cdot k \cdot d^{4} & , d \geq d_{0}\end{cases} \\
E_{R X}(k)=E_{R X-\text { elec }}(k)=E_{\text {elec }} \cdot k
\end{gathered}
$$

Where $E_{R X \text {-elec }}$ represents the energy consumed by the internal circuit of node during receiving unit data, while $E_{T X \text {-elec }}$ represents the energy consumed by the internal circuit of node during transmitting unit data, $\mu_{f s}, \mu_{m p}$ represents the free space model signal amplification parameter and the multipath fading signal amplification parameter respectively, whose values are relate with system parameters, $E_{T X-a m p}$ represents the energy consumed by sender through the amplifier.

\section{Creation of 3D-NMEERPL Cluster}

\subsection{The Calculation of the Optimal number of Cluster Heads}

Assuming $N_{\text {live }}$ sensor nodes in the initial time are uniformly distributed in the $a \times a \times a$ space, the space is divided into $K$ clusters, the number of nodes is near to $N_{\text {live }} / K$ in each cluster, including one cluster head node and $N_{\text {live }} / K-1$ non-cluster head nodes. And then the energy consumption in cluster head node and non-cluster head node is: 


$$
\begin{gathered}
E_{C H}=m E_{\text {elec }}\left(\frac{N_{\text {live }}}{K}-1\right)+m E_{d I} \frac{N_{\text {live }}}{K}+m E_{\text {elec }}+m \mu_{m p} d_{\text {toBS }}^{4} \\
E_{\text {non-CH }}=m E_{\text {elec }}+m \mu_{f s} d_{\text {toCH }}^{2}
\end{gathered}
$$

Where $d_{t o B S}$ represents the distance from the cluster head node to the base station, $d_{t o C H}$ represents the distance from the non-cluster head node to the cluster head node. Since the distance between the non-cluster head node and the cluster head node is closer, so free-space model is used ${ }^{[14]}$. Each cluster volume in network can be approximated as $a^{3} / K$, assuming distribution density of the space is $\rho(x, y, z)$, and the cluster head is located in the center of each cluster. Then, the mean of $d_{t o C H}^{2}$ is:

$$
E\left[d_{t o C H}^{2}\right]=\iiint d_{t o C H}^{2} \rho(x, y, z) d x d y d z=\iiint\left(x^{2}+y^{2}+z^{2}\right) \rho(x, y, z) d x d y d z
$$

Assuming the coordinate of cluster node is $(x, y, z)$, and each cluster is approximated as a sphere which radius is $R$, then:

$$
\frac{a^{3}}{K}=\frac{4}{3} \pi R^{3} \Rightarrow R=\sqrt[3]{\frac{3 a^{3}}{4 k \pi}}
$$

If all intra-cluster nodes obey a uniform distribution, then (the) node density is:

$$
\rho=1 /\left(a^{3} / K\right)=K / a^{3}
$$

Then, Equation (5) can be simplified as:

$$
E\left[d_{t o C H}^{2}\right]=\rho \int_{0}^{2 \pi} d \theta \int_{0}^{\pi} \sin \varphi d \varphi \int_{0}^{R} r^{4} d r=\frac{4}{5} \pi R^{5} \rho
$$

By substituting Equation (5) and (7) into Equation (8), we can get:

$$
E\left[d_{t o C H}^{2}\right]=\frac{3}{5} \cdot\left(\frac{3 a^{3}}{4 K \pi}\right)^{\frac{2}{3}}
$$

Equation (8) will be substituted into Equation (4):

$$
E_{\text {non- } C H}=m E_{\text {elec }}+\frac{3}{5} m \mu_{f s}\left(\frac{3 a^{3}}{4 K \pi}\right)^{\frac{2}{3}}
$$

Then we devoted to calculating $E\left[d_{t o B S}^{4}\right]$, as the coordinates of the base station and cluster head are $(r a, q a, p a)$ and $(x, y, z)$ respectively, and the cluster heads obey a uniform distribution in the $a \times a \times a$ space, then the distribution density is:

$$
\rho(x, y, z)=\frac{1}{a^{3}}
$$

Considering Equation (11), we have:

$$
\begin{aligned}
E\left[d_{t o B S}^{4}\right] & =\iiint\left[(x-r a)^{2}+(y-q a)^{2}+(z-p a)^{2}\right]^{2} \rho(x, y, z) d x d y d z \\
& =\int_{0}^{a} \int_{0}^{a} \int_{0}^{a}\left[(x-r a)^{2}+(y-q a)^{2}+(z-p a)^{2}\right]^{2} \frac{1}{a^{3}} d x d y d z
\end{aligned}
$$

For conveniently, we use $d_{t o B S}$ to replace the distance between the cluster head and the base station. When the protocol is running in the first round, $d_{t o B S}$ is defined as the distance from the center of network space $(a / 2, a / 2, a / 2)$ to the base station $(r a, q a, p a)$.

$$
d_{t o B S}=\sqrt{\left(r a-\frac{a}{2}\right)^{2}+\left(q a-\frac{a}{2}\right)^{2}+\left(p a-\frac{a}{2}\right)^{2}}=a \sqrt{r^{2}+q^{2}+p^{2}-(r+q+p)+\frac{3}{4}}
$$

During the second round, since all nodes send their location information to the base station in the first round, the base station could calculate the distances from all nodes to the base station and calculate the average value among the distances:

$$
d_{\text {toBS }}=\frac{\sum_{i=1}^{N_{\text {live }}} d_{\text {itoBS }}}{N_{\text {live }}}
$$


where $d_{\text {itoBS }}$ represents the distance between a common node $i$ and the sink node.

As the energy consumption of each cluster in the unit time frame is:

$$
E_{\text {cluster }}=E_{C H}+\left(\frac{N_{\text {live }}}{K}-1\right) E_{\text {non- } C H} \approx E_{C H}+\frac{N_{\text {live }}}{K} E_{\text {non-CH }}
$$

By substituting Equation (3) and (4) into Equation (15), the total energy consumption of all clusters in the unit time frame is:

$$
E_{\text {total }}=K \times E_{\text {cluster }}=2 m E_{\text {elec }} N_{\text {live }}+m E_{\text {dI }} N_{\text {live }}+K m \mu_{m p} d_{\text {toBS }}^{4}+\frac{3}{5} N_{\text {live }} m \mu_{f s}\left(\frac{3 a^{3}}{4 K \pi}\right)^{\frac{2}{3}}
$$

By taking the derivative of $\mathrm{K}$ in equation (16) the optimal value can be obtained:

$$
K_{\text {opt }}=\sqrt[5]{\frac{5}{\frac{5}{5} \mu_{f s} N_{\text {live }}\left(\frac{3 a^{3}}{4 \pi}\right)^{\frac{2}{3}}}}
$$

By substituting Equation (12) and (14) into Equation (16), we get:

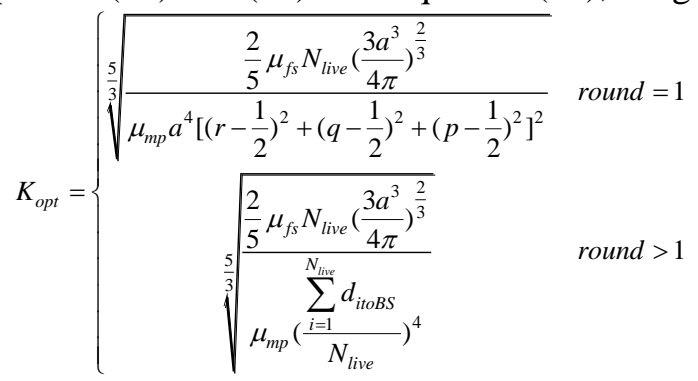

where round is the current running round, $K_{\text {opt }}$ is the optimal number of cluster heads.

\subsection{The Design of Cluster Head Selection Threshold Formula}

In addition to consider the residual energy $E_{i}$ and the distance $d s(i)$ from ordinary nodes to the sink node, in this paper, we propose the factor of node density $N(i)$ for a three-dimensional network of mobile sensor nodes, the density factor is determined by calculating the number of surviving nodes within the communication radius of each node after moving in each round. The more surviving nodes it contains, the greater the density factor of this node, where the density factor $N(i)$ is defined as $N(i)=\frac{\operatorname{Neighnor}(i)-1}{\operatorname{Neighnor}(i)}$.

Neighnor $(i)=\left\{d_{i j} \leq R, S(i) . c=S(j) . c\right\}$, where Neighnor $(i)$ is the number of neighbor nodes of node $i, R$ is the communication radius of $i$, the value of $R$ is determined by the size of the detective network space and the distance from sink node to the detection area. Then the formula of cluster head selection threshold in 3D-NMEERPL protocol is:

$$
T(n)=\frac{K_{\text {opt }}}{N_{\text {live }}-K_{\text {opt }} \times\left[r \bmod \left(N_{\text {live }} / K_{\text {opt }}\right)\right]} \cdot \frac{E_{i}-E_{\text {ave }}}{\left|E_{i}-E_{\text {ave }}\right|} \cdot \frac{E_{i}}{E_{\text {ave }}} \cdot \frac{d_{\max }}{d s(i)} \cdot N(i)
$$

When $E_{i}-E_{\text {ave }}=0$, Equation (19) can be simplified:

$$
T(n)=\frac{K_{\text {opt }}}{N_{\text {live }}-K_{\text {opt }} \times\left[r \bmod \left(N_{\text {live }} / K_{\text {opt }}\right)\right]} \cdot \frac{E_{i}}{E_{\text {ave }}} \cdot \frac{d_{\max }}{d s(i)} \cdot N(i)
$$

From the equation (19), we can see that the formula of cluster head selection threshold in 3D-NMEERPL comprehensively takes the residual energy, the average energy of the network, the distance from ordinary node to the sink node, and the node density into account. The cluster head selection algorithm has more advantage than the traditional clustering protocol. 


\section{The Multi-hop intra-cluster Routing Protocol in 3D-NMEERPL}

Within each cluster in the network, the multi-hop mode is used in data transmission from one node to another, and the number of hops is controlled within two hops, which meets the idea of reducing too much hops in multi-hop routing ${ }^{[15]}$. Firstly, a cluster is seen as a sphere whose center is the cluster head. Assuming the coordinates of cluster head is $\left(x_{0}, y_{0}, z_{0}\right)$, and the coordinates of non-cluster head node $i$ is $\left(x_{i}, y_{i}, z_{i}\right)$. Then this cluster is divided into 8 blocks, a specific block is shown in Figure 2:

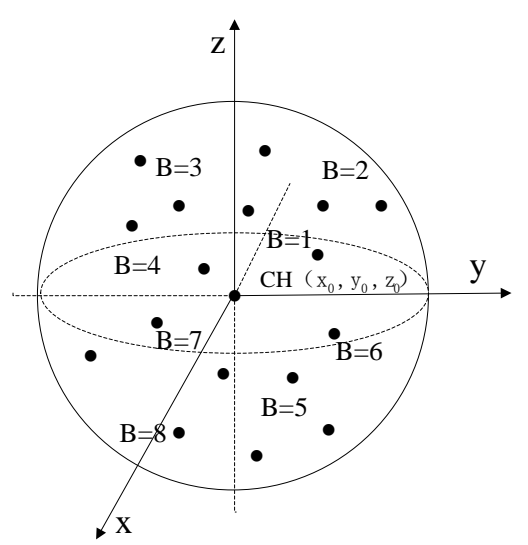

Figure 2. Routing Block in Cluster

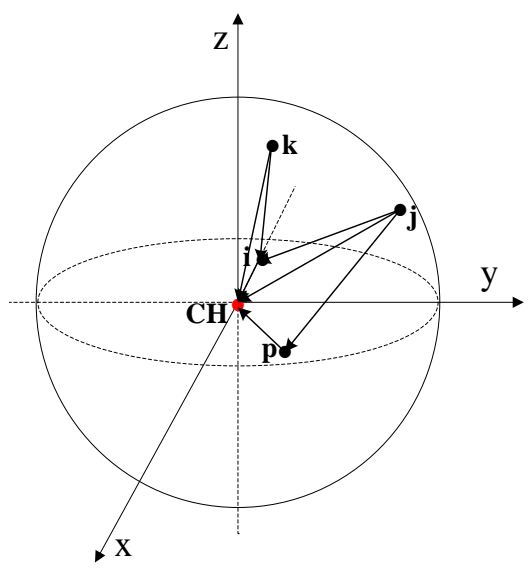

Figure 3. Selection of in-Cluster Nodes Hops

The nodes that are far away from cluster head node can only choose the node within the same block as their intermediate forwarding node in the next hop, as shown in Figure 3.

Take Figure 3 as anexample, the distance between node $\mathrm{j}$ and node $\mathrm{i}$ is $d_{j i}$, and:

$$
\begin{gathered}
E_{i} \geq E_{j} \\
d_{i}^{2}+d_{j i}^{2}<d_{j}^{2}
\end{gathered}
$$

If meeting the above two conditions, then the next hop node of $j$ is the node $i$. If the number which is in line with the next hop node is at least 2, as shown in the Figure 3: node $\mathrm{p}$ meet the requirements of the next hop meanwhile, a node has the shortest distance will be selected as the next hop node. i.e. Compare the result of $d_{i}^{2}+d_{j i}^{2}$ and $d_{p}^{2}+d_{j p}^{2}$, if $d_{i}^{2}+d_{j i}^{2}<d_{p}^{2}+d_{j p}^{2}$, then node $\mathrm{j}$ will be selected as the next hop node of node $\mathrm{i}$.

\section{Simulation Results and Analysis}

\subsection{Simulation Environment and Parameters}

The conditions of Network topology and simulation data adopted by the protocol simulation are shown in Table 1[16], network space:cube of $100 \times 100 \times 100,100$ network nodes, the nodes initial energy $1 \mathrm{~J}$.

Table. 1. Simulation Parameter

\begin{tabular}{|c|c|c|c|}
\hline Parameter & Values & Parameter & Values \\
\hline Distance Threshold & $s q r t\left(E_{f s} / E_{m p}\right)$ & $\varepsilon_{\text {amp }}$ & $100 \mathrm{pJ} / \mathrm{bit} / \mathrm{m}^{2}$ \\
\hline$E_{c \ell p c}$ & $50 \mathrm{~nJ} / \mathrm{bit}$ & $l$ & $4000 \mathrm{bits}$ \\
\hline$E_{D A}$ & $50 \mathrm{~nJ} / \mathrm{bit} / \mathrm{signal}$ & $E_{f s}$ & $10 \mathrm{pJ} / \mathrm{bit} / \mathrm{m}^{2}$ \\
\hline$E_{m p}$ & $0.0013 \mathrm{pJ} / \mathrm{bit} / \mathrm{m}^{2}$ & & \\
\hline
\end{tabular}




\subsection{Analysis}

5.2.1. Comparison of the Performance using different Protocols under the same Speed of Mobile Node: When 3D-NMEERPL, 3D-LEACH-D and 3D-DEEC [17] are running in network respectively, and the speed of mobile node is same, it is to evaluate the protocol performance by measuring the first node death time(First Node Dead, FND), the half node death time(Half Node Dead, HND) and the last node death time in network[16]. We set of sensor nodes moving speed $\mathrm{V}$ as 3 , the number of remaining nodes are shown in Figure 4 with different protocols, the total remaining energy changes at different times as shown in Figure 5 in networks.

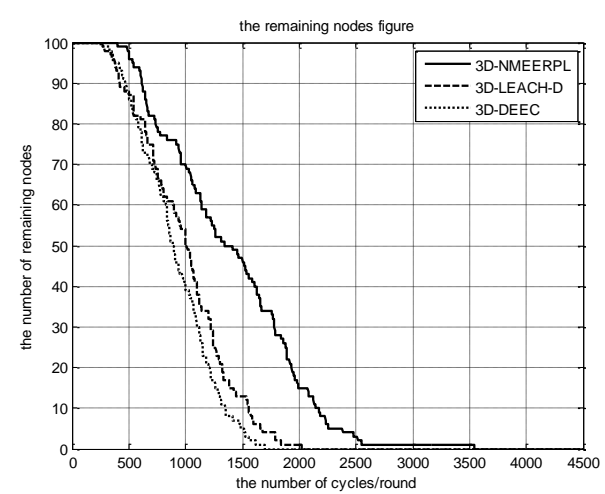

Figure 4. The remaining Nodes Change Over Time

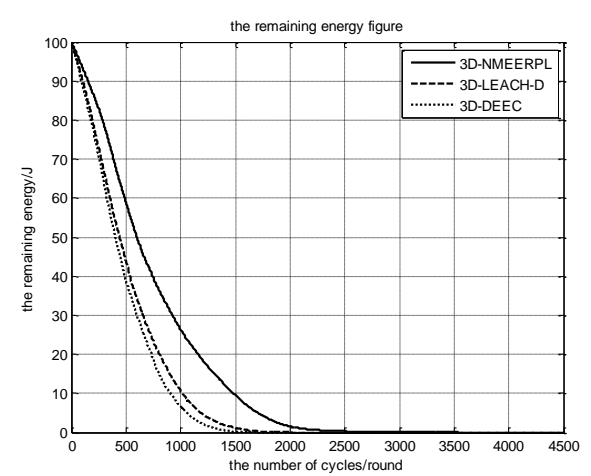

Figure 5. The Remaining Total Energy Changes Over Time

Figure 4 shows that the first node death of 3D-DEEC is in the 253th round, the half node death of 3D-DEEC is in the 888th round, the last node death of 3D-DEEC is in the 1697 th round; the first node death of 3D-LEACH-D is in the 270th round, the half node death of 3D-LEACH-D is in the 1100th round, the last node death of 3D-LEACH-D is in the 2020th round; the first node death of 3D-NMEERPL is in the 400th round, the half node death of 3D-NMEERPL is in the 1448th round, the last node death of 3D-NMEERPL is in the 3550th round. From the Figure 5, we can see 3D-NMEERPL has more remaining energy than 3D-LEACH-D and 3D-DEEC when running at the same time. 


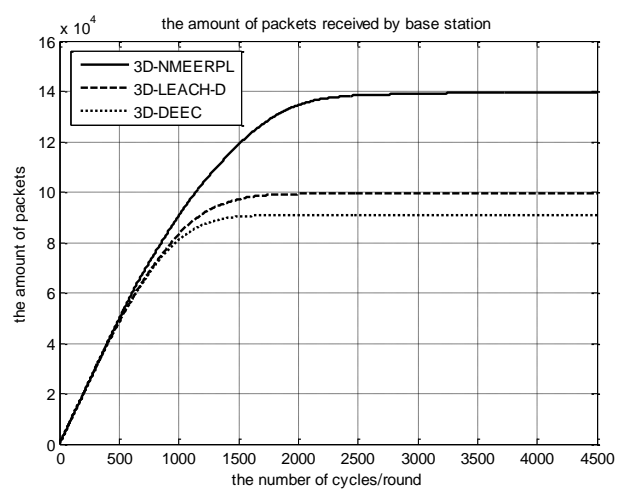

Figure 6. The Amount of Packets Received by Base Station Change Over Time

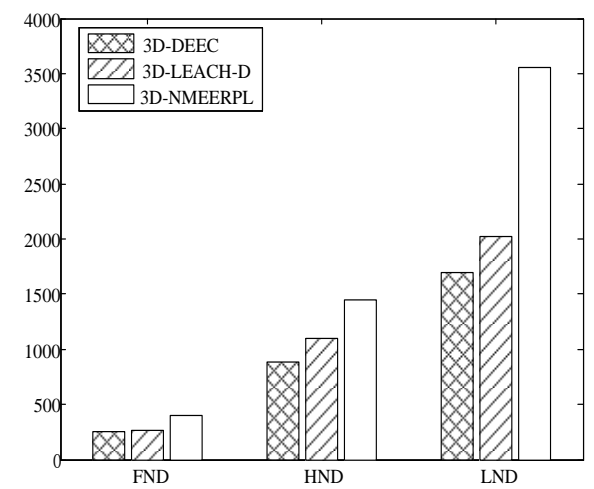

Figure 7. Comparing to Different Protocols Life Cycle

When 3D-NMEERPL, 3D-LEACH-D and 3D-DEEC are running in network respectively, the amount of packets received by base station along with the change of time curve is shown in Figure 6, the amount of forward packets through 3D-NMEERPL are more than the other two protocols.

Figure 7 shows the intuitive comparison of FND, HND and LND about the three protocols. The histogram of each group according to the sequence from left to right for 3D-DEEC, 3D-LEACH-D and 3D-NMEERPL is survival time. As can be seen from the Figure 7, the survival time of 3D-NMEERPL is significantly longer than 3D-DEEC and 3D-LEACH-D. Comparing to 3D-DEEC, 3D-NMEERPL's FND, HND and LND are extended by $58 \%, 63 \%$ and $109.2 \%$ respectively; Comparing to 3D-LEACH-D, 3D-NMEERPL's FND, HND and LND are extended by $48 \%, 31.6 \%$ and $75.7 \%$ respectively. The experimental results obtained from the data show that 3D-NMEERPL has a better energy saving effect than 3D-DEEC and 3D-LEACH-D.

5.2.2. Comparing to the performance of 3D-NMEERPL under different speed of nodes: We simulate the network performance with the moving speed $\mathrm{V}=3,5,7$ respectively. After applying 3D-NMEEERPL, the number of nodes remained along with different cycles of operation is shown in Figure8. Figure 9 shows the residual energy of nodes after different cycles of operation. 


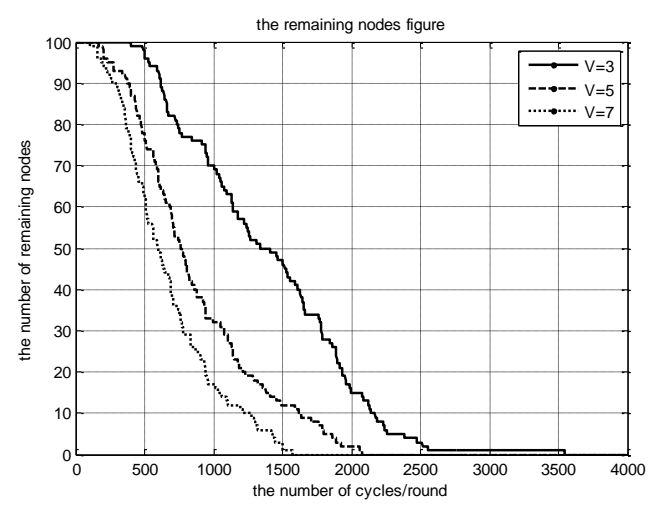

\section{Figure 8. The Remaining Number of Nodes Changes Over Time Under Different Speed}

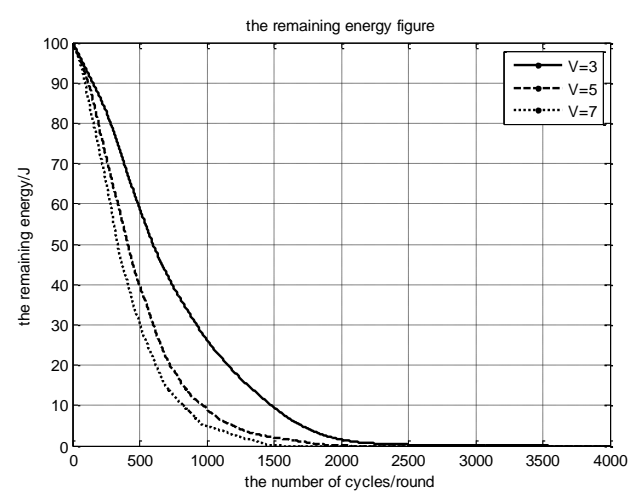

\section{Figure 9. The Network Residual Energy Changes Over Time Under Different Speed}

As shown in Figure 8, after setting the moving speed of nodes $V=3$, the time when the first node dead is the 564th round, the time when half the number of nodes dead is the 1465th round; and all the nodes dead in the 2508th round; when the moving speed is set as 5,the first node dead in the 157th round, half number of nodes dead in the736th round and all the nodes dead after 2194th round; when the moving speed V is 7, the time of the first node, half number nodes and all the nodes dead are the 96th round, 473th round and 1461 th round respectively. As we can see from the experimental results, the energy consumption is proportional to the moving speed. But the residual energy and network lifetime is inversely proportional to the moving speed

Upon operating the 3D-NMEERPL, the residual energy changes with different moving speed when the other parameters of the network are the same as shown in Figure 9. It is easy to find that the remaining energy of the network when the moving speed $\mathrm{V}=3$ is much more than that when $\mathrm{V}=5$ after the protocol runs 250 round. Also the remaining energy of the network when $\mathrm{V}=5$ is much more than that when $\mathrm{V}=7$ as well. This indicate that the faster the nodes move, the more energy they consume.

It is clearly that the total residual energy decreased when the moving speed of nodes increased with the operation of 3D-NMEERPL. Figure 10 gives a visualized comparison of FND, HND and LND with different moving speed in 3D-NMEERPL. 


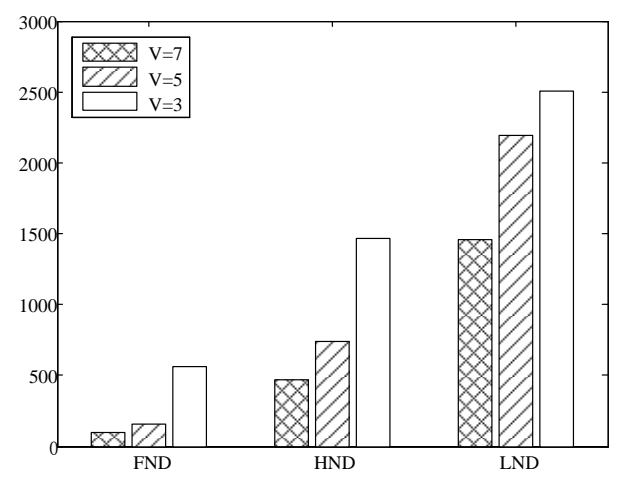

Figure 10. Comparing to the Life Cycle under Different Speed Change Over Time

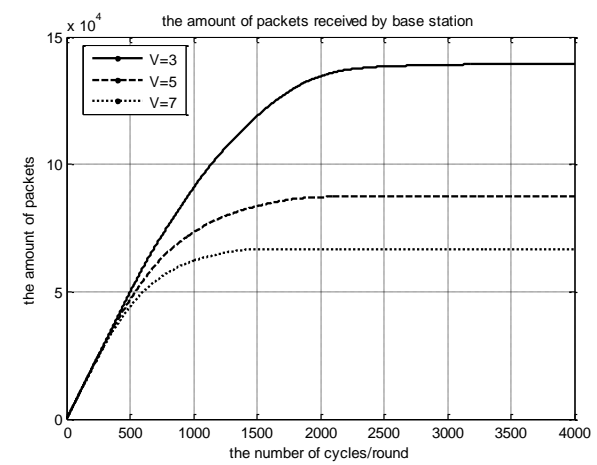

Figure 11. The Amount of Packets Received by Base Station

As shown in Figure 10, when the moving speed $\mathrm{V}=3$ the lifetime of 3D-NMEERPL is longer than that $\mathrm{V}=5$ and the lifetime when moving speed $\mathrm{V}=7$ is the shortest. So we can say that the faster the nodes move, the more energy the nodes consume and the shorter the network maintain. According to Figure 11, it's clear that the amount of packets received by the base station differ with different moving speed in 3D-NMEERPL.

When the random moving speed of sensor nodes is $V=3$, the amount of forwarding packets by base station is more than that speed $V=5$, The amount of packets forwarded by the base station decrease when the moving speed $\mathrm{V}=3,5$ and 7 respectively.

Data transmission is the important measure to evaluate the performance of routing algorithm. Wireless sensor network is a data-centric network which aims to send data to the terminal as much as possible with a certain energy consumption. As shown in Figure 6, after operating the 3D-NMEERPL, the base station forward much more packets than that of 3D-LEACH-D and 3D-DEEC. So it is rational to say that the 3D-NMEERPL has a better ability of data transmission.

\section{Conclusions}

In this paper, we present the 3D-NMEERPL protocol with the idea of clustering to optimize the number of cluster head. The algorithm considers the impacts of residual energy, distance from common node to base station and the distribution density of nodes on algorithm performance. Upon the simulation of network energy consumption, network lifetime and the amount of data packets that base station receives, it's obviously that the 3D-NMEERPL protocol outperforms the 3D-LEACH-D and 3D-DEEC in the items of network energy consumption and lifetime. 


\section{Acknowledgments}

This work was supported by the Natural Science Foundation of Jiangsu Province (no. BK2012803), Doctoral Program of the Ministry of Education of China (no. 20113219110028), and A Project Funded by the Priority Academic Program Development of Jiangsu Higher Education Institutions (PAPD).

\section{References}

[1] W. Jiang, N. Changyong and S. Ruimin, "Priority-based target coverage in directional sensor networks using a genetic algorithm", Computers and mathematic with applications, vol. 57, (2009), pp.1915-1922.

[2] R. Dutta, S. Gupta and M. K. Das, "Efficient Statistical Clustering Techniques for Optimizing Cluster Size in Wireless Sensor Network", Procedia Engineering, vol. 38, (2012), pp. 1501-1507.

[3] R. C. Luo and O. Chen, "Mobile sensor node deployment and asynchronous power management for wireless sensor networks", IEEE Transactions On Industrial Electronics, vol. 5, no. 59, (2012), pp. 2377-2385

[4] H. Ming, L. Wen-hui and C. Guo-hua, "Topology of mobile underwater wireless sensor networks", Control and Decision, vol. 12, no. 28, (2013), pp. 1761-1770.

[5] Z. S. Feng, X. Zhiliang and W. Xiaobei, "Research on keeping connectivity of group mobility", China Science Paper, vol. 7, no. 8, (2013), pp. 599-606.

[6] M. Yajie, G.Yike and G. Moustafa, "RECA: Referenced energy-based CDS algorithm in wireless sensor networks", International Journal of Communication Systems, vol. 1, no. 23, (2010), pp. 125-138.

[7] X. Ying, H. Yanglong and Z. Gang, "Geographic routing protocol for pseudo three-dimensional wireless sensor networks", Computer Engineering and Applications, vol. 22, no. 49, (2013), pp. 63-67.

[8] K. Tao, S. Hui and L. Jun-yan, "A Wireless Sensor Network Routing Algorithm Based on 3D Cell Space", Journal of Electronics \& Information Technology, vol. 6, no. 35, (2013), pp. 1298-1304.

[9] Z. Sai1, W. Wen-yong and T. Yong, "Routing Algorithm of Wireless Sensor Network Based on Entropy Standard in Dynamic Water Environment", Journal of University of Electronic Science and Technology of China, vol. 1, no. 43, (2014), pp. 82-87.

[10] Z. Jun-qiang, W. Ru-chuan and H. Hai-ping, "Research on Cluster-based Data Aggregation for Wireless Multimedia Sensor Networks", Journal of Electronics \& Information Technology, vol. 1, no. 36, (2014), pp. 8-14.

[11] T. Chao, N. Jianwei, L. Xiang, "Survey on Mobility Model”, Computer Science, vol. 10, no. 36, (2009), pp. $5-10$

[12] W. R. Heinzelman, A. Chandrakasan and H. Balakrishnan, "An Appli- cation Specific Protocol Architecture for Wireless Microsensor Networks", IEEE Transactions on Wireless Communications, vol. 4, no. 1, (2002), pp. 660-670

[13] S. MacLean and S. Datta, "Energy Constrained Positioning in Mobile Wireless Ad hoc and Sensor Networks", The 4th International Conference on Ambient Systems, Networks and Technologies, Hannover, Germany: Procedia Computer Science, vol. 19, (2013), pp. 321-329.

[14] H. Meinei, Z. Zengwei and Z. Xiaowei, "Research overview of MWSN and routing protocol", Application Research of Computers, vol. 11, no. 26, (2009), pp. 4010-4013.

[15] V. Gulhane and L. G. Malik, "Investigate Efficiency \& Reliability of Various Reprogramming Architectures for Wireless Sensor Network: A Survey", International Journal of Sensors Wireless Communications and Control, vol. 3, no. 2, (2012), pp. 184-205.

[16] W. Hao, L. Chuang and R. Fengyuan, "QoS Architecture in Wireless Sensor Network", Chinese Journal Of Computers, vol. 3, no. 32, (2009), pp. 432-440.

[17] L. Li and W. Xiangming, "Energy Efficient Optimization of Clustering Algorithm in Wireless Sensor network", Journal of Electronics \& Information Technology, vol. 4, no. 30, (2008), pp. 965-969.

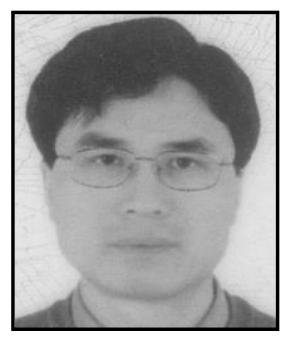

\section{Authors}

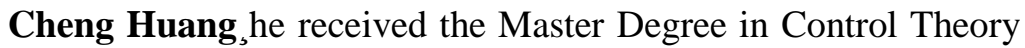
and Control Engineering from Nanjing University of Science and Technology in 2003. His current research interests on wireless sensor network, high precision detection technology, embedded system. 